\title{
An X-ray view of the active nucleus in NGC $4258^{\star}$
}

\author{
W. Pietsch ${ }^{1}$ and A. M. Read ${ }^{1,2}$ \\ 1 Max-Planck-Institut für extraterrestrische Physik, 85741 Garching, Germany \\ 2 School of Physics \& Astronomy, University Birmingham, Birmingham B15 2TT, UK \\ Received 20 November 2001 / Accepted 16 January 2002
}

\begin{abstract}
XMM-Newton observed the Seyfert 1.9 galaxy NGC 4258 in December 2000. At energies above $2 \mathrm{keV}$ a hard nuclear point source is resolved that can be fitted by a highly absorbed power-law spectrum $\left(N_{\mathrm{H}}=\right.$ $(8.0 \pm 0.4) \times 10^{22} \mathrm{~cm}^{-2}$, photon index $\left.1.64 \pm 0.08\right)$ with an unabsorbed luminosity of $7.5 \times 10^{40} \mathrm{erg} \mathrm{s}^{-1}$ in the $(2-$ 10) $\mathrm{keV}$ band. No narrow iron $\mathrm{K} \alpha$ emission line is detected (90\% upper limit of equivalent width $E W \sim 40 \mathrm{eV}$ ). The nuclear emission flux was observed to remain constant over the observation. A short archival Chandra observation taken in March 2000 further constrains the hard emission to a point source coincident with the radio nucleus. A point source $\sim 3^{\prime \prime}$ southwest of the nucleus does not contribute significantly. Spectral results of the Chandra nuclear source are comparable (within the limited statistics) to the XMM-Newton parameters. The comparison of our iron line upper limit with reported detections indicates variability of the line $E W$. These results can be explained by the relatively low nuclear absorption of NGC 4258 (which is in the range expected for its intermediate Seyfert type) and some variability of the absorbing material. Reflection components as proposed to explain the large iron line $E W$ of highly absorbed Seyfert 2 galaxies and/or variations in the accretion disk are however imposed by the time variability of the iron line flux.
\end{abstract}

Key words. galaxies: individual: NGC 4258 - galaxies: Seyfert - X-rays: galaxies

\section{Introduction}

NGC 4258 (M106) is a $B_{\mathrm{T}}=8.5$ mag nearby (distance of $7.2 \mathrm{Mpc}$, i.e. $1^{\prime \prime} \widehat{=} 35 \mathrm{pc}$, Herrnstein et al. 1999), highly inclined $\left(72^{\circ}\right.$, Tully 1988$)$ SABbc spiral spectroscopically classified as a 1.9 Seyfert galaxy (Ho et al. 1997). The strong polarization of the relatively broad optical emission lines (Wilkes et al. 1995) further supports the existence of an obscured active nucleus in NGC 4258. Water maser line emission of rotating gas near the center of NGC 4258 is modeled by a $3.6 \times 10^{7} M_{\odot}$ mass within $0.13 \mathrm{pc}$ of the nucleus, indicating a massive nuclear black hole inside a highly inclined $\left(83^{\circ}\right)$ thin gaseous disk (Miyoshi et al. 1995; Greenhill et al. 1995a,b).

Turner \& Ho (1994) detected a very bright radio continuum nuclear source, showing, in observations from March 1985 through to May 1990, long term time variability by $47 \%$ both at 6 and $20 \mathrm{~cm}$ (Hyman et al. 2001).

In X-rays ROSAT PSPC and HRI observations of NGC 4258 (Pietsch et al. 1994; Cecil et al. 1995; Vogler \& Pietsch 1999) resolved diffuse emission mostly connected to the "anomalous" arms and emission from NGC 4258 point sources. However, due to the soft energy band

Send offprint requests to: W. Pietsch, e-mail: wnp@mpe.mpg.de

* Based partly on observations with XMM-Newton, an ESA Science Mission with instruments and contributions directly funded by ESA Member States and the USA (NASA).
(0.1-2.4 keV) only upper limits could be derived for emission from the nucleus. ASCA observations of NGC 4258 were of reduced spatial resolution but extended the energy coverage to $10 \mathrm{keV}$ with improved spectral resolution. Makishima et al. (1994) reported, as well as soft emission components, a hard, highly absorbed point-like component that could be modeled by an absorbed power-law $\left(N_{\mathrm{H}} \sim 1.5 \times 10^{23} \mathrm{~cm}^{-2}\right.$, photon index $\sim 1.78$, unabsorbed luminosity $4 \times 10^{40} \mathrm{erg} \mathrm{s}^{-1}$ in the $(2-10) \mathrm{keV}$ band) and an iron $\mathrm{K}$ emission line with an equivalent width of $(250 \pm 100)$ eV. Further ASCA observations improved on the spectral parameters of the hard component, confirming a narrow iron $\mathrm{K} \alpha$ emission line at $\left(6.45_{-0.07}^{+0.10}\right) \mathrm{keV}$ with an equivalent width of $\left(107_{-37}^{+42}\right) \mathrm{eV}$, and showing time variability of the (5-10) $\mathrm{keV}$ flux and probably also the absorbing column of the hard component (Reynolds et al. 2000). BeppoSAX observations extended the spectral coverage of the power-law component to $70 \mathrm{keV}$ (Fiore et al. 2001). Wilson et al. (2001) have recently reported the results of a Chandra observation optimized to investigating the X-ray emission from the "anomalous arms". The region around the nucleus showed two compact sources, a bright heavily absorbed source coincident with the radio nucleus and the nuclear $\mathrm{H}_{2} \mathrm{O}$ maser source, and a second fainter source offset to the SW by 2 ". 5 . While the nuclear source is heavily piled-up, debilitating the determination of reliable spectral parameters, the spectrum of 


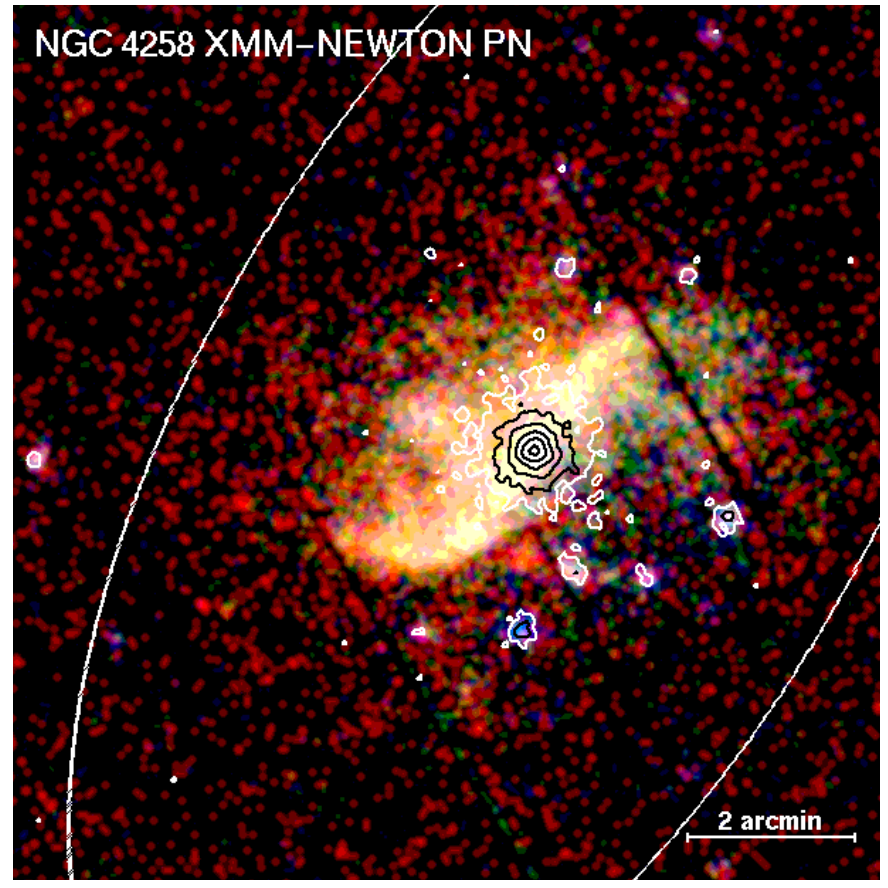

Fig. 1. Logarithmically-scaled, three-color XMM-Newton EPIC PN image of the NGC 4258 disk and nuclear regions. Red, green and blue show respectively the ROSATequivalent $(0.2-0.5) \mathrm{keV},(0.5-0.9) \mathrm{keV}$ and $(0.9-2.0) \mathrm{keV}$ bands, while the hard (2-10) $\mathrm{keV}$ emission is shown superimposed as black/white contours at levels increasing by factors of 3 from $0.12 \mathrm{ct} \operatorname{arcsec}^{-2}$. The data in each energy band have been smoothed with a PSF-equivalent Gaussian of $F W H M 5^{\prime \prime}$. The inclination-corrected optical $D_{25}$ ellipse of NGC 4258 is marked.

the weaker source is well described by an absorbed powerlaw $\left(N_{\mathrm{H}} \sim 2 \times 10^{21} \mathrm{~cm}^{-2}\right.$, photon index $\sim 1.5$, unabsorbed luminosity $5 \times 10^{38} \mathrm{erg} \mathrm{s}^{-1}$ in the $(0.5-4.5) \mathrm{keV}$ band), and is most likely an X-ray binary within NGC 4258 itself.

An XMM-Newton observation of NGC 4258 was carried out to investigate the different emission components. In this paper we will concentrate on the hard X-ray emission from the active nucleus and defer an analysis of the NGC 4258 diffuse emission and extra-nuclear point sources to a later paper. We also analyzed a short Chandra ACIS-S observation from the Chandra archive adding to the nuclear point source interpretation of the hard emission.

\section{XMM-Newton observations and results}

NGC 4258 was observed with XMM-Newton (Jansen et al. 2001) on December 8/9, 2000 during orbit 183 . The observations with the EPIC PN (Strüder et al. 2001) and MOS (Turner et al. 2001) detectors were of $\sim 16.5 \mathrm{ks}$ and $\sim 20.6$ ks respectively, with the PN in Extended Full Frame mode (thin filter) and MOS1 and MOS2 both in Full Frame mode (medium filter). Calibrated EPIC event files were created with the XMM-Newton SAS (Science Analysis Software) version 20010512_0642. X-ray events corresponding to patterns 0-4 (singles and doubles) were

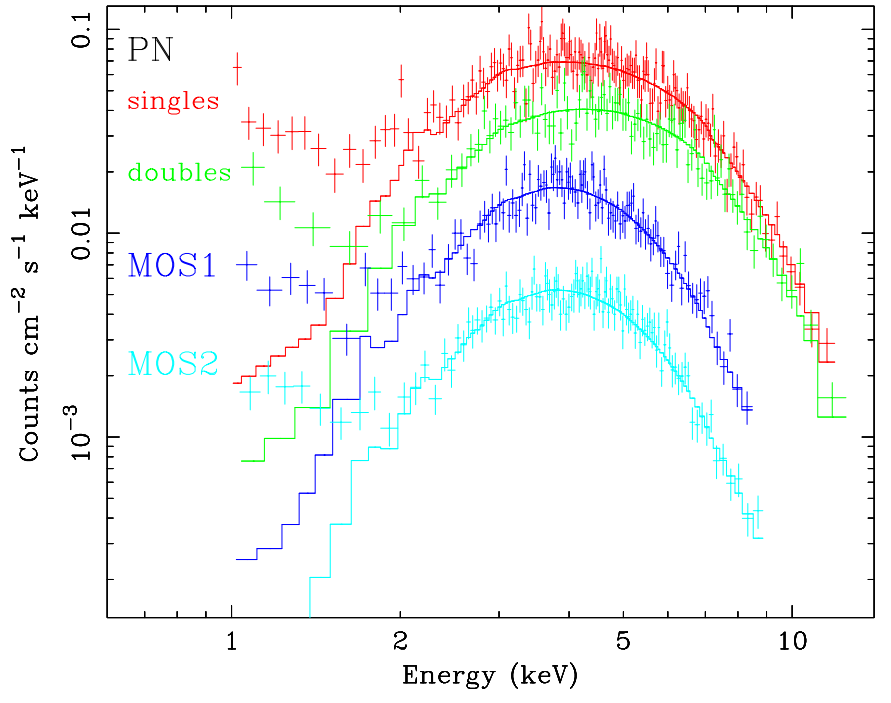

Fig. 2. XMM-Newton EPIC background-subtracted spectra of the nuclear area of NGC 4258 (extraction radius $15^{\prime \prime}$ ) for energies above $1 \mathrm{keV}$ integrated during low background times with an absorbed power-law model (see text) indicated. PN singles, PN doubles, MOS1, and MOS2 data were fitted simultaneously for energies above $2 \mathrm{keV}$. MOS1 and MOS2 intensities were shifted down for clarity by 0.5 and 1 decade, respectively.

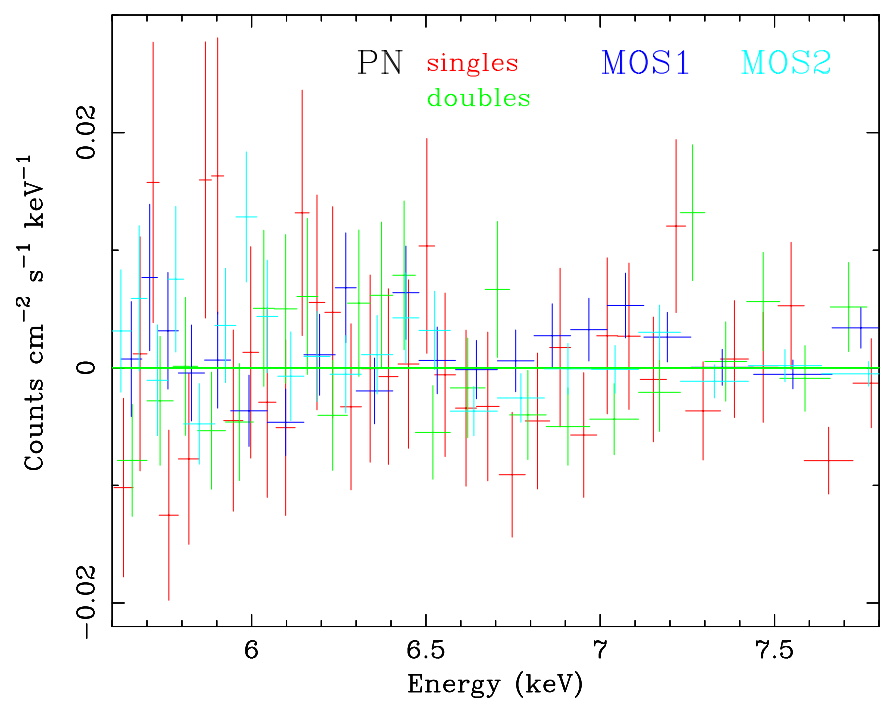

Fig. 3. Residuals of data versus power-law model of the spectral fit to the nuclear area of NGC 4258 (see Fig. 2) in the energy band around the iron $\mathrm{K} \alpha$ line energy $(5.5-7.8 \mathrm{keV})$ for EPIC PN singles and doubles, MOS1 and MOS2, respectively.

then selected for the PN camera for spectra and images; for the MOS cameras patterns 0-12 were used. Known hot or bad pixels were removed during screening. High background times at the end of the observation were rejected from the spectral fitting analysis, reducing usable integration times for spectra to $13.8,19.9$, and $20.0 \mathrm{ks}$ for PN, MOS1 and MOS2, respectively.

An EPIC PN color image of the NGC 4258 nucleus and inner disk area was created with contours of the $2-10 \mathrm{keV}$ emission superimposed (Fig. 1). Red, green and blue show respectively the ROSAT-equivalent $(0.2-0.5) \mathrm{keV}$, 
$(0.5-0.9) \mathrm{keV}$ and $(0.9-2.0) \mathrm{keV}$ bands. The image is dominated by complex unresolved emission in the soft bands with an extent of at least $6^{\prime}$ and a bright nuclear point source in the hard band. In addition there are several fainter point-like sources distributed over the NGC 4258 disk.

To characterize the nuclear emission, we extracted PN spectra from different extraction radii separately for single events and double events. For MOS1 and MOS2 spectra, we added together single, double, triple and quadruple events. As expected from Fig. 1, the contribution of the extended emission to the soft band strongly decreases for smaller extraction radii. Due to the different spectral shape, the soft contributions clearly separate from the harder nuclear emission and do not contribute above $\sim 2 \mathrm{keV}$. We therefore restricted our spectral modeling to energies above this threshold. Figure 2 shows spectra integrated for the different EPIC detectors and pattern selections using a source extraction radius of $15^{\prime \prime}$ which covers $\sim 80 \%$ of the integrated point spread function PSF (Aschenbach et al. 2000), and background spectra from a $50^{\prime \prime}$ radius area free of extended emission $\sim 4$.2 to the NE of the nucleus. For clarity, the MOS1 and MOS2 intensities have been shifted down by 0.5 and 1 decade respectively. Spectra were binned to a minimum of 30 counts per bin in order to have sufficient statistics in the individual bins for $\chi^{2}$ minimum fitting. For spectral fits we used the most recent response matrices for PN singles and doubles (epn_ff20_sY9_thin.rmf and epn_ff20_dY9_thin.rmf, Mar 2001) and MOS1 and MOS2 (m1_medv9q19t5r4_all_15.rsp and m2_medv9q19t5r4_all_15.rsp, Feb. 2001). If not quoted otherwise errors are at the $90 \%$ confidence level (e.g. $\Delta \chi^{2}=2.7$ for one interesting parameter).

A highly absorbed power-law model $\left(N_{\mathrm{H}}=(8.0 \pm\right.$ $0.4) \times 10^{22} \mathrm{~cm}^{-2}$, photon index $\left.\Gamma=1.64 \pm 0.08\right)$ yielded an acceptable fit (reduced $\chi^{2}=0.98$ for 336 degrees of freedom, d.o.f.; see Fig. 2). The normalization factors of $1.10 \pm 0.04$ and $1.14 \pm 0.04$ between MOS1 and $\mathrm{PN}$ and MOS2 and PN respectively, are caused by differences in the PSF of the instruments as well as absolute calibration uncertainties. The resulting absorbed and unabsorbed fluxes in the hard $(2-10 \mathrm{keV})$ band are 7.6 and $12.1 \times 10^{-12} \mathrm{erg} \mathrm{cm}^{-2} \mathrm{~s}^{-1}$ respectively, corresponding to luminosities of 4.7 and $7.5 \times 10^{40} \mathrm{erg} \mathrm{s}^{-1}$. As one might expect due to the limited energy range, a hard thermal bremsstrahlung model was equally acceptable $\left(N_{\mathrm{H}}=\right.$ $7.4 \times 10^{22} \mathrm{~cm}^{-2}, k T=21.7 \mathrm{keV}$, reduced $\chi^{2}=0.95$ for 336 d.o.f.).

The residuals of the data versus the power-law model in the energy band around the iron K $\alpha$ line energy (5.5$7.8 \mathrm{keV}$, see Fig. 3) do not support any emission line in this energy range, specifically not a narrow iron line around $6.45 \mathrm{keV}$ as reported by ASCA. From the XMMNewton data we determined the $90 \%$ upper limit for the photon flux of a narrow emission line around $6.45 \mathrm{keV}$ to be $4.2 \times 10^{-6}$ photons $\mathrm{cm}^{-2} \mathrm{~s}^{-1}$ (corresponding to an equivalent width of $\sim 40 \mathrm{eV}$ ). This upper limit is much lower than the values reported from the ASCA detections.
We searched for time variability of the nuclear source in the $2-10 \mathrm{keV}$ band. To do so we extracted EPIC light curves of the nuclear source with a time resolution of $200 \mathrm{~s}$. There is no long term trend within the observation. We also find no flux changes greater than $\sim 20 \%$ from the observation average on shorter time scales down to our sampling of $200 \mathrm{~s}$.

\section{Chandra observations and results}

Data (cleaned event files, images and aspect offset files) from a short exposure Chandra ACIS-S observation of NGC 4258, performed in March 2000, were obtained from the Chandra Data Archive (http://asc.harvard.edu/cgi-gen/cda). No flaring in the data was observed, and so the data over the full integration time (1205s) were used to create images and spectra.

Figure 4 shows raw ACIS-S images in the (left) soft band (below $2 \mathrm{keV}$ ) and the (right) hard band (above $2 \mathrm{keV}$ ). The images are $30^{\prime \prime}$ to a side, equivalent to the $30^{\prime \prime}$ diameter extraction circles used in the creation of the XMM EPIC NGC 4258 nuclear spectra.

A dominant source is detected at $\alpha=12^{\mathrm{h}} 18^{\mathrm{m}} 57^{\mathrm{s}} 53$, $\delta=+47^{\circ} 18^{\prime} 14^{\prime \prime} .7$ (J2000), lying $0^{\prime \prime} 7$ from the NGC 4258 central radio source of Turner \& Ho (1994), and is very prominent in the hard band image. Though it is also visible in the soft band image (though less so), what is most striking is the appearance of a soft companion $\approx 3^{\prime \prime}$ to the southwest. This companion is just about a factor of 2 fainter than the nuclear source in the soft band, whereas in the hard band it is fainter by a factor of more than 100 .

As this short Chandra observation had not been optimised to study the "anomalous arms" (as had the longer observation presented in Wilson et al. 2001), no significant pile-up is seen from the nuclear source, and the Chandra spectrum from the NGC 4258 nucleus can be studied for the first time. Programs within the Chandra data analysis suite CIAO were used to extract a spectrum from a 2 .'0625 radius circle about the position of the bright source. Similarly, a background spectrum was extracted from a source-free $9^{\prime \prime} 375$ radius circle some $13^{\prime \prime}$ away to the northeast. Once response matrix files and ancillary response files were created (again using CIAO), the spectra were binned to a minimum of 30 counts per bin. Fitting of an absorbed power-law model to the data between 1 and $10 \mathrm{keV}$ (see Fig. 5) gives rise to results entirely consistent with those obtained with XMM EPIC: $N_{\mathrm{H}}=$ $(7.2 \pm 1.8) \times 10^{22} \mathrm{~cm}^{-2}, \Gamma=1.4 \pm 0.5$, with a reduced $\chi^{2}$ of 1.36. The large errors are due to the poor statistics obtained over such a short integration time. Note the $\sim 1 \sigma$ suggestion of an iron-like feature at around $6.5 \mathrm{keV}$.

Assumption of this model gives rise to absorbed and unabsorbed hard band, (2-10) keV fluxes of 1.44 and $2.12 \times 10^{-11} \mathrm{erg} \mathrm{cm}^{-2} \mathrm{~s}^{-1}$ respectively, corresponding to luminosities of 0.89 and $1.32 \times 10^{41} \mathrm{erg} \mathrm{s}^{-1}$. 

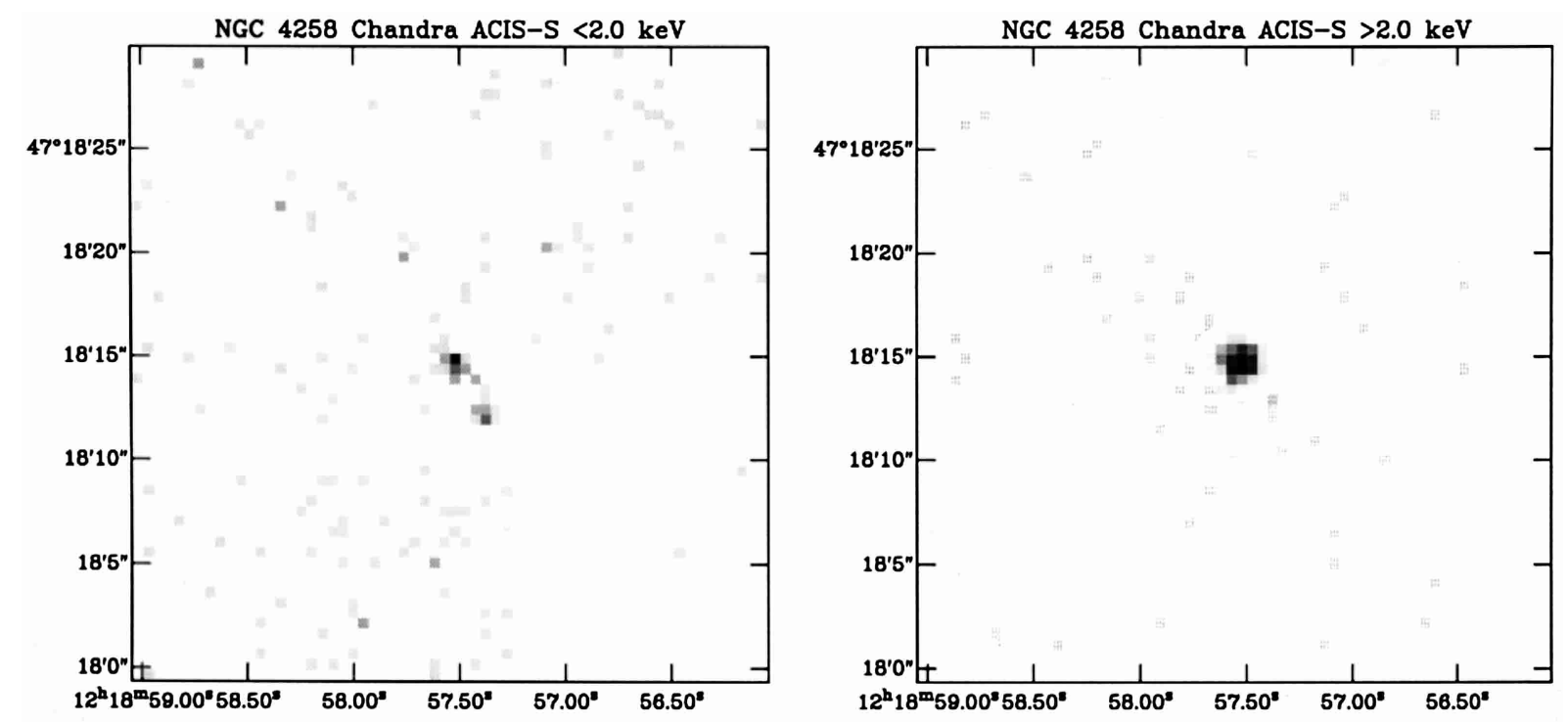

Fig. 4. Linearly-scaled, grey-scale Chandra ACIS-S images of the NGC 4258 nuclear region. The images (RA, Dec J2000.0) were binned with a pixel size of 0.5 in the soft $(<2 \mathrm{keV}$, left) and hard $(>2 \mathrm{keV}$, right) band and have maxima of 6 and 109 counts per pixel respectively.

NGC 4258 Chandra ACIS-S

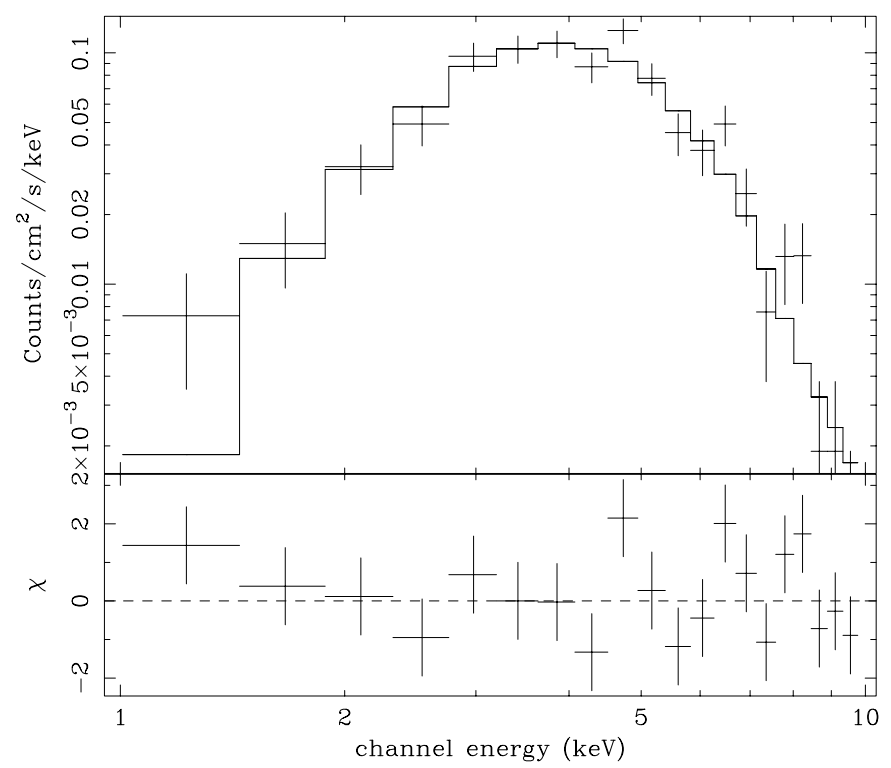

Fig. 5. Chandra background-subtracted spectrum of the nuclear area of NGC 4258 (extraction radius 2.'0625) with an absorbed power-law model (see text) indicated.

\section{Discussion}

ASCA observations of NGC 4258 established a heavily absorbed hard component in the overall X-ray spectrum which was attributed to the nucleus and confirmed by BeppoSAX (Makishima et al. 1994; Fiore et al. 2001). The ASCA and BeppoSAX observations of the hard emission of NGC 4258 however, suffered from instrument PSFs of more than an arcminute, and only higher resolution instruments such as XMM-Newton and Chandra were thought able to confirm a nuclear origin to the hard emission. Even the XMM-Newton observations do not resolve the nuclear emission from that of another reasonably bright nearby point source, most likely an Xray binary, that is resolved by Chandra (see Sect. 3). Wilson et al. (2001), analysing a deeper Chandra observation, report this source to be at a projected distance from the nucleus of 2.5 ( $87 \mathrm{pc}$ ) and characterize its spectrum as an absorbed power-law $\left(N_{\mathrm{H}}=\right.$ $\left(2.0_{-1.1}^{+1.2}\right) \times 10^{21} \mathrm{~cm}^{-2}, \Gamma=1.49_{-0.37}^{+0.50}$, absorption corrected luminosity $5.1 \times 10^{38} \mathrm{erg} \mathrm{s}^{-1}$ ), similar in slope but much less absorbed than the nuclear spectrum. Fortunately, the source is rather faint (less than $1 \%$ in flux) compared to the nuclear emission and cannot significantly influence the XMM-Newton results. Also we detect no other hard bright point-like sources within an arcmin of the nucleus that could significantly contribute to the ASCA and BeppoSAX emission, which therefore really has to originate from the nucleus.

In Table 1 we give an historical record of the NGC 4258 nuclear spectrum. The XMM-Newton power-law spectral parameters are within the range of the other measurements. The photon index is on the hard side but compatible within the errors of the ASCA results. Only the BeppoSAX index pointed at a somewhat softer spectrum. The absorbing column $\left(\sim 8 \times 10^{22} \mathrm{~cm}^{-2}\right)$ seems to have not varied significantly since the first ASCA measurements that pointed at an absorption a factor 1.52 higher. The luminosity however, seems to have varied on a timescale of several years by a factor of about two starting from low values in 1993 with a maximum in 1996 to 1998. The XMM-Newton measurements indicate that in December 2000 the nuclear luminosity was again at a lower level. Fiore et al. (2001) report variability of a factor of about 2 on half-day timescales as well as smaller scale variations $(10 \%-20 \%)$ on timescales as short as $1 \mathrm{hr}$. During the XMM-Newton observations the source did not show any variability at similar 
Table 1. Historical high energy spectral data for NGC 4258.

\begin{tabular}{|c|c|c|c|c|c|c|c|c|}
\hline Observatory & Date & $\begin{array}{c}N_{\mathrm{H}} \\
{\left[10^{22} \mathrm{~cm}^{-2}\right]}\end{array}$ & $\Gamma$ & $\begin{array}{l}E_{\text {line }} \\
{[\mathrm{keV}]}\end{array}$ & $\begin{array}{c}W_{\text {line }} \\
{[\mathrm{eV}]}\end{array}$ & $f_{\mathrm{X}}^{*}$ & $L_{\mathrm{X}}^{* *}$ & Ref. \\
\hline ASCA & May 1993 & $15 \pm 2$ & $1.78 \pm 0.29$ & $6.5 \pm 0.2$ & $250 \pm 100$ & & 4.2 & 1 \\
\hline ASCA & May 1993 & $13.6_{-2.2}^{+2.1}$ & $1.78_{-0.26}^{+0.22}$ & & & 5.1 & & 2 \\
\hline ASCA & May 1996 & $9.2 \pm 0.9$ & $1.71_{-0.17}^{+0.18}$ & & & 8.3 & & 2 \\
\hline ASCA & Jun. 1996 & $8.8_{-0.6}^{+0.7}$ & $1.83 \pm 0.13$ & & & 8.8 & & 2 \\
\hline ASCA & Dec. 1996 & $9.7 \pm 0.8$ & $1.87 \pm 0.15$ & & & 9.5 & & 2 \\
\hline BeppoSAX & Dec. 1998 & $9.4 \pm 1.2$ & $2.11 \pm 0.14$ & $6.57 \pm 0.20$ & $85 \pm 65$ & & 10 & 3 \\
\hline ASCA & May 1999 & $9.5_{-0.9}^{+2.1}$ & $1.86_{-0.13}^{+0.40}$ & & & 4.0 & & 2 \\
\hline ASCA & May 1999 & $8.9_{-0.7}^{+0.4}$ & $1.83_{-0.09}^{+0.06}$ & $6.45_{-0.07}^{+0.10}$ & $107_{-37}^{+42}$ & & 5.7 & 2 \\
\hline Chandra & Mar. 2000 & $7.2 \pm 1.8$ & $1.4 \pm 0.5$ & & & & 13 & this work \\
\hline XMM-Newton & Dec. 2000 & $8.0 \pm 0.4$ & $1.64 \pm 0.08$ & 6.45 & $<40$ & & 7.5 & this work \\
\hline
\end{tabular}

Notes and references: * $5-10 \mathrm{keV}$ uncorrected flux $\left[10^{-12} \mathrm{erg} \mathrm{cm}^{-2} \mathrm{~s}^{-1}\right] .^{* *} 2-10 \mathrm{keV}$ absorption corrected luminosity for NGC 4258 distance of $7.2 \mathrm{Mpc}\left[10^{40} \mathrm{erg} \mathrm{s}^{-1}\right]$. (1) Makishima et al. (1994); (2) Reynolds et al. (2000); (3) Fiore et al. (2001).

amplitudes. With its absorption-corrected (2-10) keV luminosity of typically $7 \times 10^{40} \mathrm{ergs}^{-1}$, the AGN in NGC 4258 is presently not very active compared to other Seyfert galaxies. Risaliti et al. (1999) report luminosities from Seyfert galaxies ranging from $3 \times 10^{40} \mathrm{erg} \mathrm{s}^{-1}$ to $2 \times 10^{44} \mathrm{erg} \mathrm{s}^{-1}$.

The high X-ray absorption of the nuclear component in NGC 4258 is expected, given the optically-derived Seyfert 1.9 classification of the galaxy, using the so-called "unified model" (Antonucci 1993). This model assumes that the engine is at work in all active galactic nuclei and differences between types 1 and 2 AGN are ascribed solely to orientation effects, i.e. that our line of sight is (type 2) or is not (type 1) obscured by optically thick material. Risaliti et al. (1999) showed that most "strict" Seyfert 2 nuclei are highly obscured $\left(N_{\mathrm{H}}>10^{23} \mathrm{~cm}^{-2}\right)$ or even Compton thick $\left(N_{\mathrm{H}}>10^{24} \mathrm{~cm}^{-2}\right)$. Intermediate type 1.8-1.9 Seyfert galaxies on the other hand are characterized by an average $N_{\mathrm{H}}$ much lower than "strict" Seyfert 2 galaxies. The absorption of the NGC 4258 nuclear spectrum of $\sim 8 \times 10^{22} \mathrm{~cm}^{-2}$ fits into this scenario.

From the first reports of hard X-ray emission from NGC 4258, a narrow iron $\mathrm{K} \alpha$ emission line was always needed to model the spectra. According to Table 1, the strongest line compared to the continuum was needed for the 1993 ASCA data. With XMM-Newton we do not detect a line, and our $E W$ upper limit is more than a factor of 6 below the Makishima et al. (1994) initial ASCA detection and still a factor of more than 2 below the later ASCA and BeppoSAX values (Reynolds et al. 2000; Fiore et al. 2001). Note that Makishima et al. (1994) report that no background was subtracted from the May 1993 ASCA raw spectra and therefore, one might have doubts regarding the line strength in this analysis if the background contains contributions from an intrinsic iron feature. It must be stated however, that the existence of the line was clearly demonstrated in the later ASCA observations. The BeppoSAX equivalent width from December 1998 is, due to the large errors, compatible with both the ASCA results from May 1999 and with our XMM-Newton upper limit from December 2000.

There are at least two possible explanations for this result: (1) The difference could be caused by the larger sky area used to accumulate the ASCA and BeppoSAX spectra. In this way an extended component from the NGC 4258 disk could be picked up that would not be covered by the much smaller spectral extraction region used here with XMM-Newton. We tried to rule out this explanation by extracting XMM-Newton spectra from a comparably large area. In these spectra we did not detect any iron line emission. The upper limit to the equivalent width however is similar to the measurements by earlier satellites. (2) The most straightforward explanation however, is that the line intensity is varying with time.

Several groups have calculated the contribution of an obscuring torus to the X-ray spectra of Seyfert galaxies using Monte Carlo simulations (e.g. Awaki et al. 1991; Leahy \& Creighton 1993; Ghisellini et al. 1994). They find strong dependence of the iron line $E W$ at $6.4 \mathrm{keV}$ on the absorbing column and geometrical factors. For the relatively low nuclear absorption in NGC $4258, E W$ s of $\sim 100 \mathrm{eV}$ or less are expected. This is compatible with our findings. These simple models therefore would be sufficient to explain the XMM-Newton data and we would not need to invoke additional reflection components. Such a component is for instance needed to explain the iron line $E W$ of $>2 \mathrm{keV}$ measured by Chandra in the Seyfert 2 nucleus of M51 (Terashima \& Wilson 2001). 
According to Table 1, the iron line varied in absolute flux by a factor of at least 2 in 18 months (from May 1999 to December 2000) setting an upper limit on the size-scale of the fluorescing region of $0.5 \mathrm{pc}$. This seems to argue against a torus origin for the iron line which would imply a significantly larger size scale. Instead, it favours an origin of the line in the accretion disk much closer to the nucleus. If the line originated in the outer regions of the accretion disk as proposed by Reynolds et al. (2000) using different arguments, its variability could reflect intensity changes of the nuclear power law source (that may not have been covered directly by the observations listed in Table 1). Alternative explanations for the line variability might be changes in the disk (e.g. the "inner" edge of the optically thin disk, or the ionization structure of the disk surface).

The - up till now - sparse data on the NGC 4258 $6.4 \mathrm{keV}$ line seem to indicate a correlation between the amount of absorption and the $E W$ of the line. However, an anticorrelation of the line $E W$ with the nuclear luminosity can also not be ruled out. More high quality spectra are clearly needed to clarify the behaviour, and such results may then force revisions and improvements to the simple unification picture.

\section{Summary}

The XMM-Newton observations of the Seyfert 1.9 galaxy NGC 4258 show a hard nuclear point source with a spectrum that can be modeled by a highly absorbed power-law. The analysis of a short archival Chandra observation constrains the hard emission to a point source coincident with the radio nucleus. During the XMM-Newton observation the nuclear emission flux remained constant. Our upper limit to narrow iron $\mathrm{K} \alpha$ line emission is significantly lower than earlier detections by ASCA and BeppoSAX and indicates time variability of this component. Simple absorption models would be sufficient to explain the reported line fluxes and upper limits. The suggested long term line variability, however, seems to point at a more complex scenario including reflection from the accretion disk and/or variability of the disk geometry and ionization.

The detailed analysis of XMM-Newton data from the extranuclear point sources as well as from the diffuse emission from the "anomalous" arms and the interstellar medium in the disk and halo of NGC 4258 will be published in a following paper.
Acknowledgements. We thank the referee (Christopher Reynolds) for helpful comments. The XMM-Newton project is supported by the Bundesministerium für Bildung und Forschung/Deutsches Zentrum für Luft- und Raumfahrt (BMBF/DLR), the Max-Planck Society and the HeidenhainStiftung.

\section{References}

Antonucci, R. 1993, ARA\&A, 31, 473

Aschenbach, B., Briel, U. G., Haberl, F., et al. 2000, Proc. SPIE, 4012, 731

Awaki, H., Koyama, K., Inoue, H., \& Halpern, J. P. 1991, PASJ, 43, 195

Cecil, G., Wilson, A. S., \& de Pree, C. 1995, ApJ, 440, 181

Fiore, F., Pellegrini, S., Matt, G., et al. 2001, ApJ, 556, 150

Ghisellini, G., Haardt, F., \& Matt, G. 1994, MNRAS, 267, 743

Greenhill, L. J., Henkel, C., Becker, R., Wilson, T. L., \& Wouterloot, J. G. A. 1995a, A\&A, 304, 21

Greenhill, L. J., Jiang, D. R., Moran, J. M., et al. 1995b, ApJ, 440,619

Herrnstein, J. R., Moran, J. M., Greenhill, L. J., et al. 1999, Nature, 400, 539

Ho, L. C., Filippenko, A. V., \& Sargent, W. L. W. 1997, ApJS, 112,315

Hyman, S. D., Calle, D., Weiler, K. W., et al. 2001, ApJ, 551, 702

Jansen, F., Lumb, D., Altieri, B., et al. 2001, A\&A, 365, L1

Leahy, D. A., \& Creighton, J. 1993, MNRAS, 263, 314

Makishima, K., Fujimoto, R., Ishisaki, Y., et al. 1994, PASJ, 46, L77

Miyoshi, M., Moran, J., Herrnstein, J., et al. 1995, Nature, 373, 127

Pietsch, W., Vogler, A., Kahabka, P., Jain, A., \& Klein, U. 1994, A\&A, 284, 386

Reynolds, C. S., Nowak, M. A., \& Maloney, P. R. 2000, ApJ, 540,143

Risaliti, G., Maiolino, R., \& Salvati, M. 1999, ApJ, 522, 157

Strüder, L., Briel, U., Dennerl, K., et al. 2001, A\&A, 365, L18

Terashima, Y., \& Wilson, A. S. 2001, ApJ, 560, 139

Tully, R. B. 1988, Nearby galaxies catalog (Cambridge and New York, Cambridge University Press)

Turner, J. L., \& Ho, P. T. P. 1994, ApJ, 421, 122

Turner, M. J. L., Abbey, A., Arnaud, M., et al. 2001, A\&A, $365, \mathrm{~L} 27$

Vogler, A., \& Pietsch, W. 1999, A\&A, 352, 64

Wilkes, B. J., Schmidt, G. D., Smith, P. S., Mathur, S., \& McLeod, K. K. 1995, ApJ, 455, L13

Wilson, A. S., Yang, Y., \& Cecil, G. 2001, ApJ, 560, 1 